(pp 66-74)

\title{
Relation between Fatty Infiltration of Cervical Extensor Muscles and Postural Stability in Patients with Cervical Spondylotic Radiculopathy
}

\author{
Tsubasa MITSUTAKE, PT, MS, Yuji CHUDA, PT \\ Department of Rehabilitation, Shiroishi Kyoritsu Hospital \\ Tsuyoshi OISHI, MD \\ Department of Neurosurgery, Shiroishi Kyoritsu Hospital \\ Tsubasa MITSUTAKE, PT, MS, Etsuo HORIKAWA, PhD \\ Graduate School of Medicine, Saga University
}

Purpose: This study aimed to clarify the association of muscle fat infiltration with cervical extensor muscle laterality and postural stability in patients with cervical spondylotic radiculopathy.

Methods: We evaluated 20 patients with cervical spondylotic radiculopathy. The measure of the amount of fat within the muscles was obtained by the ratio of the pixel intensities of the upper trapezius and cervical multifidus muscles to the pixel intensity of a standardized region of intermuscular fat using magnetic resonance imaging. In the stabilometer test, postural sway determined by a shift in the center of pressure was measured in the upright position on a platform with the subject's eyes closed for $60 \mathrm{~s}$. We used two parameters for evaluation: locus length (LNG) and environmental area (EA). Spearman's rank and partial correlation test was used to analyze the association between fat indices in the upper trapezius and multifidus muscles and the results of the LNG. To determine whether any factor could the LNG, a step-wise multiple regression analysis was performed with LNG as the dependent variable and age, gender, body mass index, neck disability index and muscle fat infiltration with cervical extensor muscle laterality as independent variable.

Results: Impaired cervical multifidus muscle had a significant correlation with LNG. The multiple regression analysis demonstrated that muscle fat infiltration with impaired cervical multifidus muscle was significant contributors to LNG, accounting for $62 \%$ of the variability.

Conclusion: In the patients with cervical spondylotic radiculopathy, muscle fat infiltration with impaired cervical multifidus muscle possibly affected postural control. 